\title{
Overexpression of miR-506 suppresses proliferation and promotes apoptosis of osteosarcoma cells by targeting astrocyte elevated gene-1
}

\author{
JIE YAO $^{1,2^{*}}$, LI QIN $^{3 *}$ SEN MIAO $^{2}$, XIANGSHAN WANG $^{2}$ and XUEJIAN WU ${ }^{3}$ \\ ${ }^{1}$ Orthopedics Department, The First Affiliated Hospital of Zhengzhou University; ${ }^{2}$ Spine Department 3 , \\ Zhengzhou Orthopedics Hospital; ${ }^{3}$ Orthopedics Department 3, The First Affiliated Hospital of Zhengzhou University, \\ Zhengzhou, Henan 450052, P.R. China
}

Received April 21, 2015; Accepted June 10, 2016

DOI: $10.3892 / \mathrm{ol} .2016 .4827$

\begin{abstract}
There is increasing evidence that microRNAs (miRs) are implicated in tumor development and progression; however, their specific roles in osteosarcoma are not well understood. The aim of the present study was to investigate the role of miR-506 in the pathogenesis of osteosarcoma. The expression levels of miR-506 and astrocyte elevated gene-1 (AEG-1) mRNA were detected using quantitative polymerase chain reaction, and the protein levels of AEG-1, $\beta$-catenin, c-myc and cyclin D1 were determined using western blot analysis. The effects of miR-506 and AEG-1 on cell viability, colony forming ability and apoptosis were assessed using MTT assay, colony formation assay, and flow cytometry, respectively. Lucifer reporter assays were used to demonstrate whether AEG-1 is a direct target of miR-506. The present study identified that miR-506 was downregulated in osteosarcoma tissues and cells. Overexpression of miR-506 suppressed the proliferation and induced apoptosis in osteosarcoma cells in vitro and inhibited tumor formation in vivo. Overexpression of miR-506 significantly inhibited the luciferase activity of AEG-1 with a wild-type 3'-untranslated region, providing clear evidence that AEG-1 was a direct and functional downstream target of miR-506. Similar to the overexpression of miR-506, downregulation of AEG-1 lead to an inhibitory effect on osteosarcoma in vitro. Furthermore, overexpression of miR-506 or downregulation of AEG-1 inhibited the Wnt/ $\beta$-catenin signaling pathway, and inhibition of this pathway by $\beta$-catenin small interfering RNA or CGP049090, a small molecule inhibitor,
\end{abstract}

Correspondence to: Professor Xuejian Wu, Orthopedics Department 3, The First Affiliated Hospital of Zhengzhou University, 1 Jianshe East Road, Zhengzhou, Henan 450052, P.R. China

E-mail: xuejianwuzzu@163.com

${ }^{*}$ Contributed equally.

Key words: astrocyte elevated gene-1, miR-506, osteosarcoma, $\mathrm{Wnt} / \beta$-catenin pathway suppressed cell proliferation and induced apoptosis in vitro. Overall, the present data indicated that miR-506 functions as a tumor suppressor by targeting AEG-1 in osteosarcoma via the regulation of the $\mathrm{Wnt} / \beta$-catenin signaling pathway.

\section{Introduction}

Osteosarcoma is the most common primary bone malignancy in children and adolescents, and is characterized by a highly malignant tendency to destroy the surrounding tissues and metastasize almost exclusively to the lung, which is the primary cause of mortality among patients $(1,2)$. In young patients, osteosarcoma is most often localized in the distal femur and proximal tibia region (3). Although osteosarcoma has been treated with neoadjuvant chemotherapy in combination with surgery for more than three decades, patients with recurrent or metastatic osteosarcoma have an extremely poor prognosis, with a long-term survival rate of $<10 \%$ (4). To date, the molecular mechanisms underlying the initiation, development and metastasis of osteosarcoma are not fully elucidated, and it is essential to identify novel therapeutic targets and develop therapeutic strategies against osteosarcoma.

MicroRNAs (miRs) belong to a group of small noncoding, single-stranded RNA fragments measuring 18-25 nucleotides in length, which are critical regulators in tumorigenesis and cancer progression (5). Previously, miRs have been demonstrated to suppress translation or directly cleave target mRNA through complementary sequence pairing to the 3'-untranslated region (UTR) or coding region of target mRNA (6). These data indicate that miRs may be used as diagnostic biomarkers and may function either as oncogenes or tumor suppressors based on the effects of their target mRNAs $(7,8)$. It has been reported in osteosarcoma that multiple miRs, including miR-29, miR-125b, miR-143 and miR-199a-3p, are involved in tumor growth, progression and metastasis (9-12). A previous study revealed that restoration of miR-506 in malignant transformed human bronchial epithelial cells suppressed cell proliferation (13). In addition, overexpression of miR-506 inhibited transforming growth factor- $\beta$-induced epithelial-mesenchymal transition and suppressed the adhesion, invasion and migration of human breast cancer cells (14). Furthermore, overexpression of 
miR-506 in established hydroxycamptothecin-resistant colon cancer cells conferred resistance to hydroxycamptothecin by inhibiting the expression of peroxisome proliferator-activated receptor $\alpha$ (15). Although miR-506 has been subjected to extensive study in recent years, its role in the initiation and progression of osteosarcoma, and the molecular mechanisms by which miR-506 exerts its effects, are poorly understood.

Astrocyte elevated gene-1 (AEG-1), also known as lysine-rich carcinoembryonic antigen-related cell adhesion molecule 1 or metadherin, was initially characterized as a human immunodeficiency virus-1- and tumor necrosis factor- $\alpha$-inducible gene in primary human fetal astrocytes $(16,17)$. Although AEG-1 is ubiquitously expressed in numerous cell types, the expression level of AEG-1 is higher in certain solid tumors, including breast and prostate cancer, malignant glioma, hepatocellular carcinoma and melanoma, compared to normal counterpart tissues (18). In addition, patients with elevated AEG-1 levels have shorter overall survival times compared with patients with lower AEG-1 levels (19). Previously, certain studies have demonstrated that AEG-1 is significantly associated with chemoresistance and progression of osteosarcoma, and AEG-1 has been suggested to act as a useful biomarker for the prediction of osteosarcoma progression and prognosis (20-22). Therefore, targeted downregulation of AEG-1 may be an effective treatment strategy against osteosarcoma.

The aim of the present study was to investigate the role of miR-506 in the pathogenesis of osteosarcoma. The present results revealed that the expression of AEG-1 was significantly increased, while the level of miR-506 was significantly decreased, in human osteosarcoma tissues and cells. Overexpression of miR-506 and knockdown of AEG-1 attenuated proliferation and promoted apoptosis of osteosarcoma in vitro. Furthermore, miR-506 overexpression was demonstrated to inhibit osteosarcoma cell growth in vivo. Therefore, the present study provides evidence that miR-506 suppresses osteosarcoma development by targeting AEG-1, partly via regulating the $\mathrm{Wnt} / \beta$-catenin signaling pathway.

\section{Materials and methods}

Clinical specimens and cell culture. A total of 19 pairs of primary osteosarcoma tissues and matched adjacent non-cancerous bone tissues were obtained from the First Affiliated Hospital of Zhengzhou University (Zhengzhou, China) to identify the expression level of miR-506 using quantitative polymerase chain reaction (qPCR). The characteristics of the patients are listed in Table I. All diagnoses were determined according to the criteria of the World Health Organization (23). Written informed consent was obtained from the patients. The study was approved by the Local Research Ethics Committee of the First Affiliated Hospital of Zhengzhou University (Zhengzhou, China).

Human normal osteoblastic hFOB 1.19 and human osteosarcoma MG63 cell lines were obtained from the American Type Culture Collection (Manassas, VA, USA) and were cultured in Dulbecco's Modified Eagle Medium supplemented with $10 \%$ fetal bovine serum and $1 \%$ penicillin/streptomycin (Invitrogen $^{\mathrm{TM}}$; Thermo Fisher Scientific, Inc., Waltham, MA, USA) at $37^{\circ} \mathrm{C}$ in a humidified atmosphere containing $5 \%$ $\mathrm{CO}_{2}$.
Table I. Clinicopathological characteristics of osteosarcoma patients.

\begin{tabular}{lr} 
Characteristics & Patients, $\mathrm{n}$ \\
\hline Age, years & 16 \\
$\leq 18$ & 3 \\
$>18$ & \\
Gender & 10 \\
Male & 9 \\
Female & \\
Histology & 13 \\
Osteoblastic & 5 \\
Chondroblastic & 1 \\
Other & \\
Metastasis & 13 \\
Yes & 6 \\
No & \\
Tumor-node-metastasis stages & 7 \\
I + II & 12 \\
III + IV & \\
\hline
\end{tabular}

$q P C R$. Total RNA was extracted from frozen tissues and osteosarcoma cells using TRIzol Reagent (Invitrogen ${ }^{\mathrm{TM}}$ ), according to the manufacturer's protocol. In total, $1 \mu \mathrm{l}$ DNase (Qiagen, Inc., Valencia, CA, USA) was used. The reverse transcriptase of RNA was performed with the miScript II RT kit (Qiagen, Inc.), according to the manufacturer's protocol. The expression level of miR-506 was quantified by qPCR using TaqMan microRNA Assays (Applied Biosystems ${ }^{\mathrm{TM}}$; Thermo Fisher Scientific, Inc.). Specific primer sets were designed using Primer Premier version 5.0 software (PREMIER Biosoft, Palo Alto, CA, USA) and synthesized by Sangon Biotech Co., Ltd. (Shanghai, China) as follows: miR-506, forward 5'-GACATGCATAAGGCACCCTTC-3' and reverse 5'-GTGCAGGGTCCGAGGT-3'; AEG-1, forward 5'-AAATAGCCAGCCTATCAAGACTC-3' and reverse 5'-TTCAGACTTGGTCTGTGAAGGAG-3'; $\beta$-catenin, forward 5'-GCTGATTTGATGGAGTTGGA-3' and reverse 5'-TCAGCTACTTGTTCTTGAGTGAA-3'; and $\beta$-actin, forward 5'-TGGACTTCGAGCAGGAAATGG-3' and reverse 5'-ACG TCGCAC TTCATGATCGAG-3'. qPCR was performed on a ABI 7900 Sequence Detection System (Applied Biosystems; Thermo Fisher Scientific, Inc.) using the following cycling conditions: Denaturation, $95^{\circ} \mathrm{C}$ for $15 \mathrm{~min}$, followed by 40 cycles of $94^{\circ} \mathrm{C}$ for $15 \mathrm{sec}, 55^{\circ} \mathrm{C}$ for $30 \mathrm{sec}$ and $72^{\circ} \mathrm{C}$ for $30 \mathrm{sec}$. The relative expression of mRNA was calculated and normalized using the $\Delta \mathrm{Cq}$ method (24) relative to $\beta$-actin. Each test was performed in triplicate.

Transfection. Control miR (miR-control; 5'-UGUGCGACG CGGCUGGAUGCG-3'), hsa-miR-506 mimic (miR-506 mimic; 5'-UAAGGCACCCUUCUGAGUAGA-3'), control anti-miR (anti-miR-control; 5'-CACUACGCAGAACCGGAA UAU-3'), anti-miR-506 mimic (5'-UCUACUCAGAAGGGU GCCUUA-3') and small interfering RNA (si) targeting AEG-1 
coding sequences (si-AEG-1; forward, 5'-GACACUGGAGAU GCUAAUAUU-3' and reverse 5'-UAUUAGCAUCUCCAG UGUCUU-3') were chemically synthesized by Genepharma, Co., Ltd. (Shanghai, China). Cells $\left(5 \times 10^{5}\right.$ cells/well) were transfected with the miRs and si using Lipofectamine ${ }^{\circledR} 2000$ (Invitrogen ${ }^{\mathrm{TM}}$ ), according to the manufacturer's protocol.

Western blot analysis. Cells were lysed using RIPA Lysis and Extraction Buffer (Thermo Fisher Scientific, Inc.), and protein concentration was measured using BCA Protein Assay kit (Pierce ${ }^{\mathrm{TM}}$; Thermo Fisher Scientific, Inc.). Western blot analysis was conducted as described previously (1). Briefly, following a $48 \mathrm{~h}$ transfection, the proteins were separated by sodium dodecyl sulfate-polyacrylamide gel electrophoresis and subsequently transferred to nitrocellulose membranes (Bio-Rad Laboratories, Inc., Hercules, CA, USA). The membranes were blocked with $10 \%$ skimmed milk (Sigma-Aldrich, St. Louis, MO, USA) at room temperature for $2 \mathrm{~h}$ and incubated overnight at $4^{\circ} \mathrm{C}$ with rabbit polyclonal anti-AEG-1 (catalog no., 40-6500; 1:500; Invitrogen ${ }^{\mathrm{TM}}$ ), mouse monoclonal anti- $\beta$-catenin (catalog no., 610154; 1:500; BD Transduction Laboratories ${ }^{\mathrm{TM}}$; BD Biosciences, Franklin Lakes, NJ, USA), rabbit polyclonal anti-c-myc (catalog no., sc-764; 1:200; Santa Cruz Biotechnology, Inc., Dallas, TX, USA), mouse monoclonal anti-cyclin D1 (catalog no., sc-450; 1:100; Santa Cruz Biotechnology, Inc.), and rabbit polyclonal anti- $\beta$-actin (catalog no., 4967; 1:2,000; Cell Signaling Technology, Inc., Danvers, MA, USA) antibodies. After washing, the membranes were incubated for $2 \mathrm{~h}$ at room temperature with horseradish peroxidase-conjugated secondary immunoglobulin $\mathrm{G}$ goat anti-mouse (catalog no, sc-2005; 1:10,000) or goat anti-rabbit (catalog no, sc-2004; 1:10,000) antibodies (Santa Cruz Biotechnology, Inc.). The proteins were visualized using ImageQuant LAS4000 (GE Healthcare Life Sciences, Chalfont, UK).

MTT assay. For cell viability assays, MG63 cells were transfected with miR-506 mimic or si-AEG-1. Following transfection for $48 \mathrm{~h}$, MG63 cells were seeded in a 96-well plate at a density of $4 \times 10^{3}$ cells per well. After incubation for 24, 48, 72 and $96 \mathrm{~h}$ at $37^{\circ} \mathrm{C}$ in a humidified atmosphere containing $5 \%$ $\mathrm{CO}_{2}, 10 \mu \mathrm{l}$ MTT [5 mg/ml in phosphate-buffered saline (PBS); Sigma-Aldrich] was added to each well and the plates were incubated for a further $4 \mathrm{~h}$. After removal of the medium, each cell was treated with $150 \mu 1$ dimethyl sulfoxide to dissolve the formazan crystals. Optical density values were determined using a microplate reader (Model 680 Microplate Reader; Bio-Rad Laboratories, Inc.) at a wavelength of $490 \mathrm{~nm}$.

MG63 cells transfected with si- $\beta$-catenin (sense, 5 '-CAG UUGUGGUUAAGCUCUUdTdT-3' and antisense, 3'-dTdT GUCAACACCAAUUCGAGAA-5'; Genepharma, Co., Ltd) or treated with 0, 5 and $10 \mu \mathrm{M}$ CGP049090 (Sigma-Aldrich) were seeded in a 96-well plate and incubated for $48 \mathrm{~h}$ at $37^{\circ} \mathrm{C}$ in a humidified atmosphere containing $5 \% \mathrm{CO}_{2}$. The subsequent MTT was as aforementioned.

Colony formation assay. Subsequent to transfection for $48 \mathrm{~h}$, MG63 cells were seeded in a 6-well plate at a density of 500 cells per well and cultured for 10 days. Colony formation was viewed by staining the cells with $2 \%$ Giemsa solution
(Merck Millipore, Darmstadt, Germany) for 10 min following fixation with $10 \%$ methanol for 5 min.

Flow cytometry. Cell apoptosis was evaluated using Annexin V/fluorescein isothiocyanate (FITC) Apoptosis Detection kit (BD Biosciences) and propidum iodide (PI; Sigma-Aldrich), according to the manufacturer's protocol. Briefly, cells were harvested using $0.25 \%$ trypsin $48 \mathrm{~h}$ after transfection or treatment with CGP049090, washed twice with cold PBS, and re-suspended in binding buffer. Subsequently, cells were incubated with $5 \mu \mathrm{l}$ Annexin V/FITC and $5 \mu \mathrm{l} \mathrm{PI}$ for $15 \mathrm{~min}$ at room temperature in the dark. A flow cytometer (BD Biosciences) was used to detect apoptosis in MG63 cells.

Luciferase reporter assays. MG63 cells were seeded in 24 -well plates $24 \mathrm{~h}$ prior to transfection. Subsequently, the cells were transiently co-transfected with $0.3 \mu \mathrm{g}$ wild type or mutant reporter plasmid (Agilent Technologies, Santa Clara, CA, USA) and $50 \mathrm{nM}$ miR-506 mimic or miR-control using Lipofectamine 2000. Firefly and Renilla luciferase activities were measured $48 \mathrm{~h}$ subsequent to transfection using the Dual Luciferase Assay (Promega, Madison, WI, USA), according to the manufacturer's protocol. Firefly luciferase activity was normalized to Renilla, and the value of firefly luciferase activity/Renilla luciferase activity was analyzed. Three independent experiments were performed in triplicate.

Tumor formation in nude mice. A total of 8,4-6-week-old, male BALB/c nude mice (nu/nu; 20-25 g) were obtained from Vital River Laboratories Co., Ltd. (Beijing, China). The animals were housed under specific pathogen-free conditions and fed with chow and sterile water ad libitum in a $12 \mathrm{~h}$ light/dark cycle at $23 \pm 2{ }^{\circ} \mathrm{C}$. In total, $2 \times 10^{6} \mathrm{MG} 63$ cells stably overexpressing miR-506 mimic or miR-control were subcutaneously injected into 4 to 6 -week-old nude mice ( $n=8$ per group). Tumors were measured with calipers to estimate the tumor volume between day 7 and 28 following injection according to the following formula: Tumor volume $=0.5 \mathrm{x}$ length $\mathrm{x}$ width $^{2}$. The mice were sacrificed a total of 28 days subsequent to inoculation, and tumour weights were measured. All animal procedures were performed with the approval of the Local Medical Experimental Animal Care Commission of the First Affiliated Hospital of Zhengzhou University.

Statistical analysis. All data are presented as the mean \pm standard deviation, and analyzed using SPSS version 19.0 software (IBM SPSS, Armonk, NY, USA). The significance of the observed differences between groups was calculated using Student's t-test or one-way analysis of variance. $\mathrm{P}<0.05$ was considered to indicate a statistically significant difference.

\section{Results}

Level of miR-506 is inversely associated with AEG-1 protein expression in osteosarcoma. AEG-1 is ubiquitously expressed in numerous cell types and is overexpressed in certain solid tumors (25). To determine whether AEG-1 is overexpressed in osteosarcoma, the expression level of AEG-1 in human osteosarcoma tissues and osteosarcoma MG63 cell line was measured using western blot analysis. As shown in 

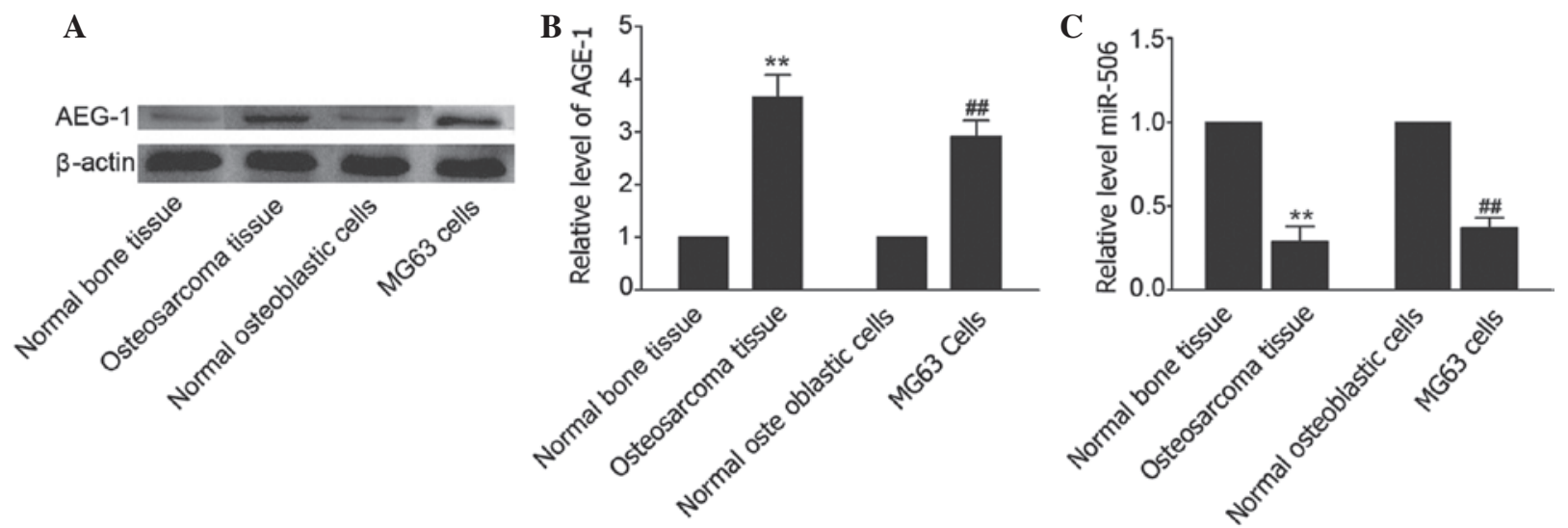

Figure 1. Levels of miR-506 were inversely associated with AEG-1 protein (64 kDa) expression in osteosarcoma tissues and human osteosarcoma MG63 cell line. (A and B) Western blot analysis revealed that the level of AEG-1 expression was increased in osteosarcoma tissues and cell lines. $\beta$-actin was used as a control. (C) Quantitative polymerase chain reaction was used to investigate mRNA expression of miR-506 in osteosarcoma tissues and MG63 cell line. ${ }^{* *} \mathrm{P}<0.01$ vs. normal bone tissue; ${ }^{\# \#} \mathrm{P}<0.01$ vs. normal osteoblastic hFOB 1.19 cells. miR, microRNA; AEG-1, astrocyte elevated gene-1.
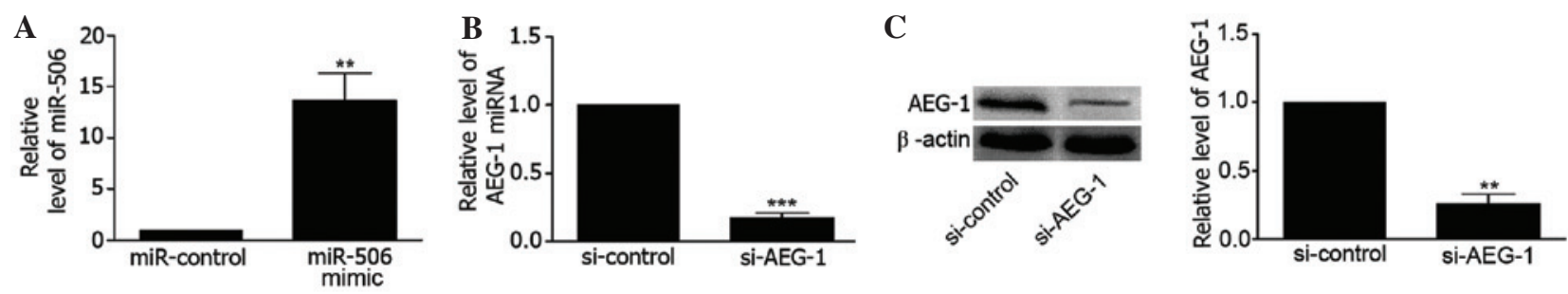

D

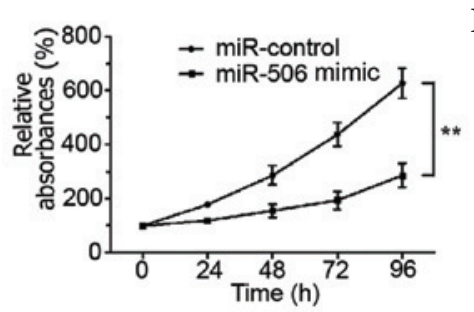

$\mathbf{F}$

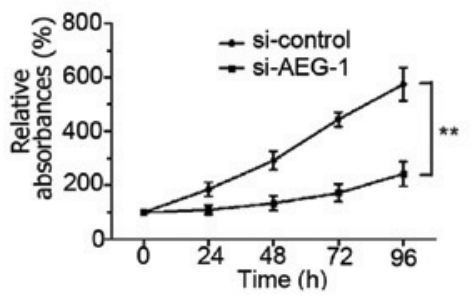

$\mathbf{E}$
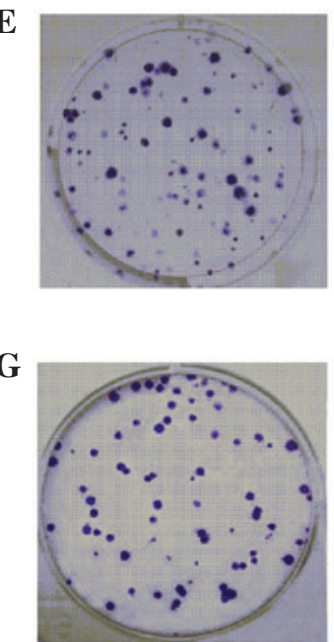
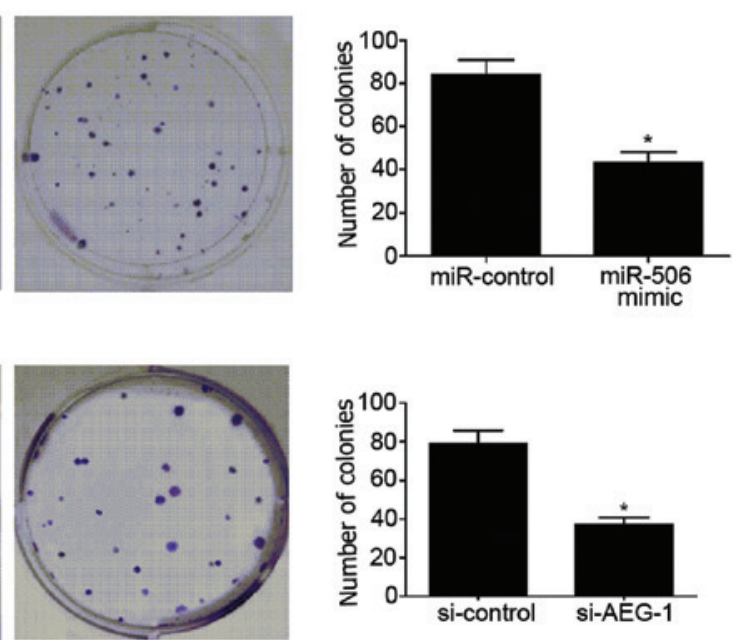

Figure 2. Upregulation of miR-506 and downregulation of AEG-1 inhibited the growth of human osteosarcoma MG63 cells. (A) qPCR revealed that the relative level of miR-506 was significantly increased in MG63 cells transfected with miR-506 mimics. ${ }^{* *} \mathrm{P}<0.01 \mathrm{vs.} \mathrm{miR-control}$. (B) qPCR showed that the relative expression of AEG-1 mRNA was decreased in MG63 cells transfected with si-AEG-1. ${ }^{* * *} \mathrm{P}<0.001$ vs. si-control. (C) Western blot analysis showed that the relative level of AEG-1 protein $(64 \mathrm{kDa})$ was decreased in MG63 cells transfected with si-AEG-1. ${ }^{* *} \mathrm{P}<0.01$ vs. si-control. (D and E) Upregulation of miR-506 (D) inhibited MG63 cell proliferation and (E) decreased the number of colonies of MG63 cells. ${ }^{*} \mathrm{P}<0.05,{ }^{* *} \mathrm{P}<0.01$ vs. miR-control. (F and G) Downregulation of AEG-1 (F) inhibited MG63 cell proliferation and $(\mathrm{G})$ decreased the number of colonies of MG63 cells. ${ }^{*} \mathrm{P}<0.05,{ }^{* * *} \mathrm{P}<0.01$ vs. si-control. miR, microRNA; AEG-1, astrocyte elevated gene-1; qPCR, quantitative polymerase chain reaction; si, small interfering RNA.

Fig. 1A and B, an elevated level of AEG-1 was observed in osteosarcoma tissues compared with matched adjacent non-cancerous tissues $(\mathrm{P}=0.0063)$. In addition, the level of AEG-1 was increased in MG63 cells compared with human normal osteoblastic hFOB 1.19 cells. TargetScan (www. targetscan.org/vert_71/) revealed that miR-506 is predicted to target the AEG-1-associated gene. To investigate the effects of miR-506 on osteosarcoma, the level of miR-506 was identified in osteosarcoma tissues and osteosarcoma MG63 cell line.
The results showed that the level of miR-506 in osteosarcoma tissues and cells was decreased compared with matched adjacent non-cancerous tissues and hFOB 1.19 cells, respectively $(\mathrm{P}=0.0090$ and $\mathrm{P}=0.0086$, respectively; Fig. $1 \mathrm{C})$. Therefore, the present study hypothesized that miR-506 may participate in the regulation of osteosarcoma by targeting AEG-1.

Upregulation of miR-506 suppresses proliferation of osteosarcoma cells. To clarify the regulatory effects of miR-506 
A

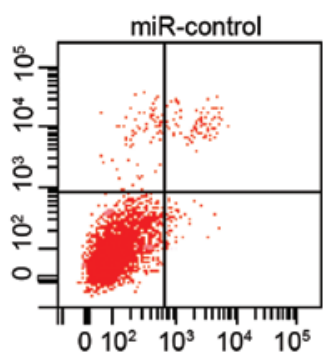

B

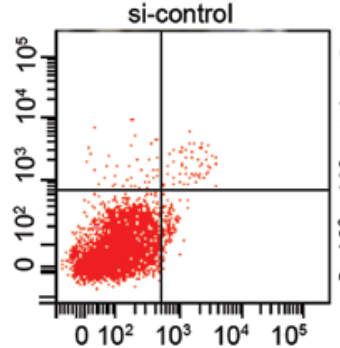

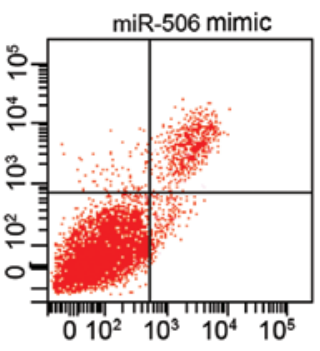
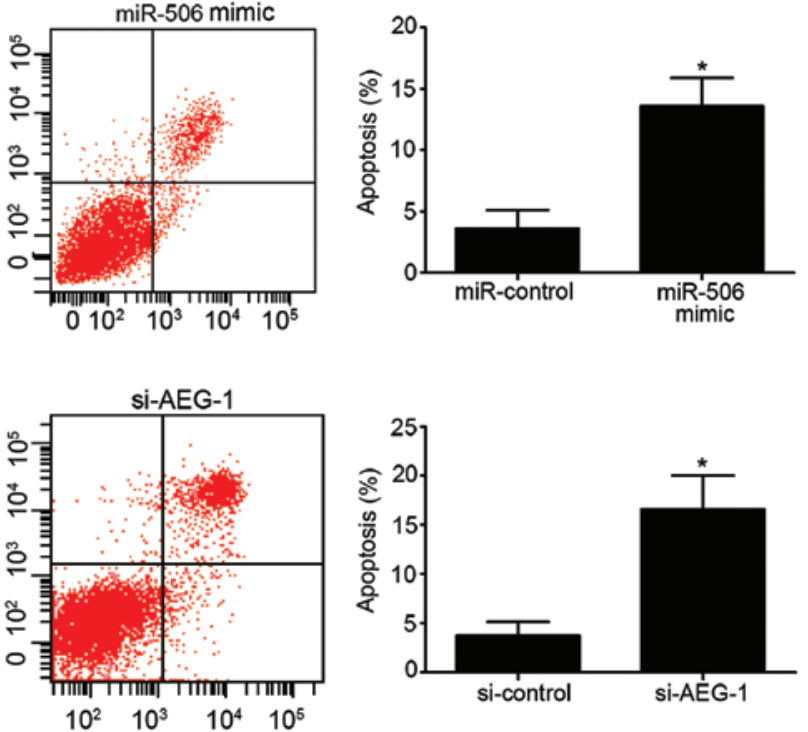

Figure 3. Effects of miR-506 and AEG-1 on the apoptosis of human osteosarcoma MG63 cells. (A) Overexpression of miR-506 enhanced the apoptosis of MG63 cells. " $\mathrm{P}<0.05$ vs. miR-control. (B) Knockdown of AEG-1 induced the apoptosis of MG63 cells. "P<0.05 vs. si-control. miR, microRNA; AEG-1, astrocyte elevated gene-1; si, small interfering RNA.

on osteosarcoma, MG63 cells were transfected with miR-506 mimics. As shown in Fig. 2A, miR-506 was overexpressed in MG63 cells ( $\mathrm{P}=0.0094)$, as determined by qPCR. In addition, the mRNA and protein level of AEG-1 was downregulated in MG63 cells by transfection with si-AEG-1 ( $\mathrm{P}=0.0003$ and $\mathrm{P}=0.0013$, respectively; Fig. 2B and C). Overexpression of miR-506 significantly decreased the viability of MG63 cells compared with the miR-control-transfected group of cells $(\mathrm{P}=0.0038$; Fig. 2D) and inhibited the colony forming ability of the cells $(\mathrm{P}=0.0157$; Fig. 2E). Similarly, downregulation of AEG-1 inhibited the viability of MG63 cells ( $\mathrm{P}=0.0024$; Fig. 2F), and inhibited the colony forming ability of the cells ( $\mathrm{P}=0.0012$; Fig. 2G). These findings suggest that miR-506 and AEG-1 are involved in the regulation of MG63 cell proliferation.

Upregulation of miR-506 inhibits apoptosis of osteosarcoma cells. The present study additionally assessed the effects of miR-506 and AEG-1 on the apoptosis of MG63 cells. Overexpression of miR-506 significantly increased the apoptotic rate of MG63 cells compared to the miR-control-transfected group ( $\mathrm{P}=0.0265$; Fig. 3A). Similarly, downregulation of AEG-1 induced a higher apoptotic rate of MG63 cells compared with the si-control group ( $\mathrm{P}=0.0137$; Fig. 3B). Overall, these results indicate that overexpression of miR-506 and downregulation of AEG-1 have a clear ability to induce MG63 cell apoptosis.

AEG-1 is directly targeted by miR-506 in MG63 cells. Bioinformatics analysis using TargetScan suggested that miR-506 was a predicted to target AEG-1, and the 'seed sequence' of miR-506 matched the 3'-UTR of the AEG-1 mRNA (Fig. 4A). To confirm AEG-1 was directly targeted and regulated by miR-506 in MG63 cells, luciferase reporter genes were constructed using the AEG-1 3'-UTR and the mutant counterpart at the miR-506 binding regions, and these were co-transfected with miR-506 mimics or miR-control into MG63 cells. Overexpression of miR-506 significantly inhibited the luciferase activity of AEG-1 with the wild-type 3'-UTR ( $\mathrm{P}=0.0018)$, but not with mutant 3'-UTR (Fig. 4B). To further determine whether miR-506 could functionally affect the expression of AEG-1, the present study determined if the expression level of AEG-1 was regulated by miR-506. The results demonstrated that overexpression of miR-506 suppressed the expression of AEG-1 in MG63 cells, and downregulation of miR-506 increased the level of AEG-1 in hFOB 1.19 cells $(\mathrm{P}=0.0168$ and $\mathrm{P}=0.0401$, respectively; Fig. 4C and D). Overall, these results suggest that the 3'-UTR of AEG-1 is a functional target site of miR-506 in osteosarcoma cells.

miR-506 inhibits osteosarcoma cell growth in vivo. To investigate whether miR-506 has a role in a mouse model of osteosarcoma, MG63 cells stably overexpressing miR-506 or control-miR were injected subcutaneously into nude mice. The tumor volume was measured every 7 days. A total of 28 days following inoculation, mice were sacrificed and tumor weights were measured. Overexpression of miR-506 significantly inhibited the tumor growth of MG63 xenografts compared with the negative control group, since the average volume and weight of the miR-506-overexpressing tumors were notably decreased $(\mathrm{P}=0.0023$ and $\mathrm{P}=0.0017$, respectively; Fig. 5A and B). Therefore, miR-506 clearly attenuates osteosarcoma cell growth in vivo.

miR-506 inhibits osteosarcoma development via regulation

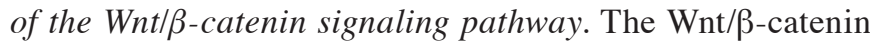
signaling pathway has been widely implicated in the development of multiple tumors, including osteosarcoma (26). The present study detected the expression levels of $\beta$-catenin, c-myc, and cyclin D1 in MG63 cells to determine whether miR-506 inhibits osteosarcoma via the $\mathrm{Wnt} / \beta$-catenin pathway. As shown in Fig. 6A, upregulation of miR-506 and downregulation of AEG-1 clearly decreased the expression 
A

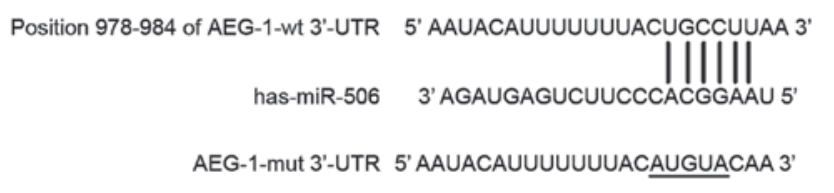

$\mathbf{C}$

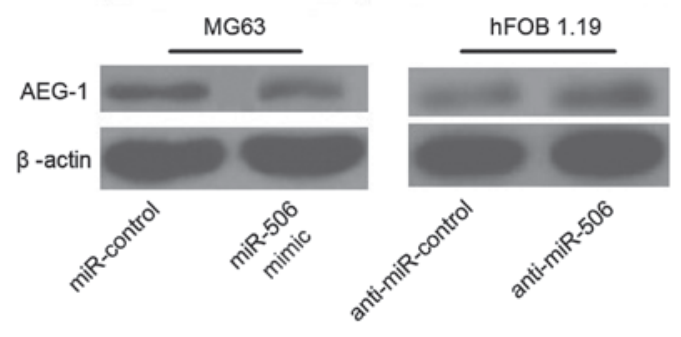

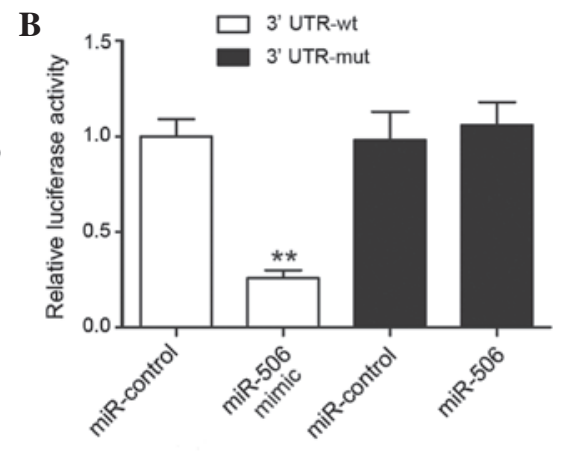

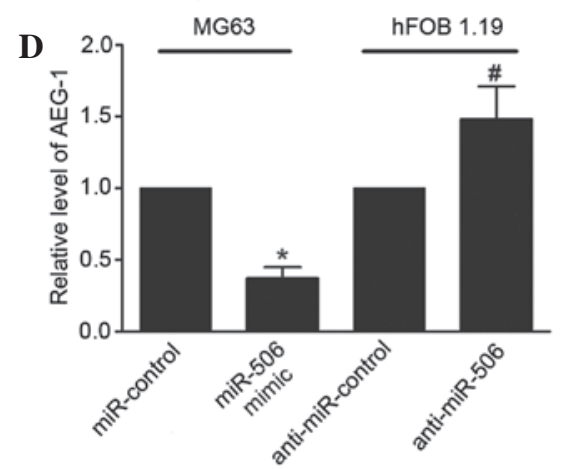

Figure 4. AEG-1 is a direct target of miR-506 in human osteosarcoma MG63 cells. (A) Specific binding locations of miR-506 and the 3'-UTR of AEG-1. (B) Luciferase reporter assay showed that the relative luciferase activity of AEG-1 3'-UTR was significantly decreased in MG63 cells co-transfected with wt or mut AEG-1 3'-UTR and miR-506 mimics. (C and D) Western blot analysis showed that the relative level of AEG-1 protein (64 kDa) was decreased in MG63 cells following transfection with miR-506 mimics, but was increased in human normal osteoblastic hFOB 1.19 cells following transfection with anti-miR-506 mimics. ${ }^{*} \mathrm{P}<0.05,{ }^{* *} \mathrm{P}<0.01$ vs. miR-control; ${ }^{*} \mathrm{P}<0.05$ vs. anti-miR-control. miR, microRNA; AEG-1, astrocyte elevated gene-1; UTR, untranslated region; wt, wild-type; mut, mutant.
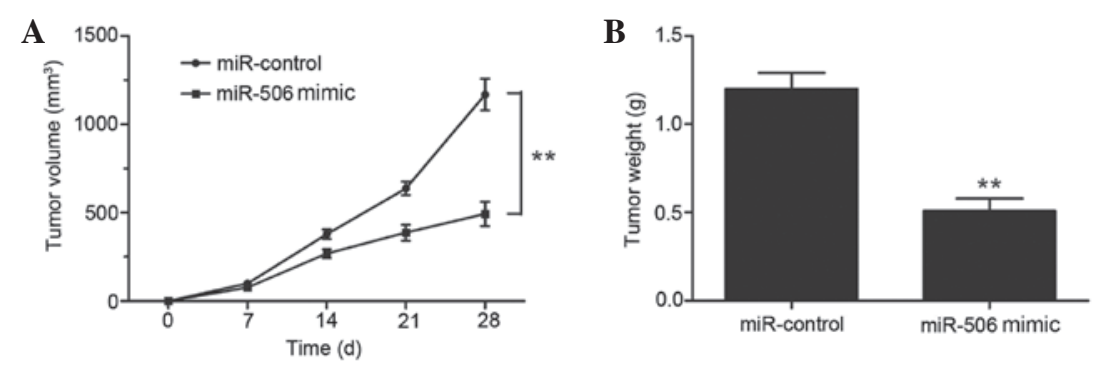

Figure 5. miR-506 inhibited the growth of human osteosarcoma MG63 xenografts. (A and B) Overexpression of miR-506 decreased tumor (A) volumes and (B) weights compared with miR-control. ${ }^{* *} \mathrm{P}<0.01$ vs. miR-control. miR, microRNA.

levels of $\beta$-catenin $(\mathrm{P}=0.0268$ and $\mathrm{P}=0.0134$, respectively), c-myc $(\mathrm{P}=0.0166$ and 0.0129 , respectively) and cyclin $\mathrm{D} 1$ $(\mathrm{P}=0.0288$ and $\mathrm{P}=0.0260$, respectively) in MG63 cells. In addition, the $\mathrm{Wnt} / \beta$-catenin signaling pathway was inhibited by the present study using si- $\beta$-catenin. The levels of $\beta$-catenin mRNA and protein were significantly decreased in MG63 cells following transfection $(\mathrm{P}=0.0011$ and $\mathrm{P}=0.0103$, respectively; Fig. $6 \mathrm{~B}$ and $\mathrm{C}$ ). Blocking of the $\mathrm{Wnt} / \beta$-catenin signaling pathway suppressed proliferation $(\mathrm{P}=0.0236)$ and induced apoptosis $(\mathrm{P}=0.0046)$ of MG63 cells (Fig. 6D and $\mathrm{E}$ ). Furthermore, CGP049090, a small molecule inhibitor of $\mathrm{Wnt} / \beta$-catenin, inhibited the expression of $\beta$-catenin $(5 \mu \mathrm{M}$, $\mathrm{P}=0.0227 ; 10 \mu \mathrm{M}, \mathrm{P}=0.0086)$, c-myc $(5 \mu \mathrm{M}, \mathrm{P}=0.0213 ; 10 \mu \mathrm{M}$, $\mathrm{P}=0.0017)$ and cyclin $\mathrm{D} 1(5 \mu \mathrm{M}, \mathrm{P}=0.0243 ; 10 \mu \mathrm{M}, \mathrm{P}=0.0033)$ in MG63 cells in a concentration-dependent manner (Fig. 6F). CGP049090 clearly inhibited proliferation (5 $\mu \mathrm{M}, \mathrm{P}=0.0373$; $10 \mu \mathrm{M}, \mathrm{P}=0.0088)$ and induced apoptosis $(\mathrm{P}=0.0008)$ of MG63 cells (Fig. 6G and $\mathrm{H}$ ). These data demonstrate that
miR-506 suppresses osteosarcoma by regulation of AEG-1 through inhibition of the $\mathrm{Wnt} / \beta$-catenin signaling pathway.

\section{Discussion}

To improve osteosarcoma therapy, novel therapeutic targets require identification, and therapeutic strategies need to be developed. Recently, the utilization of miRs has provided novel insights into osteosarcoma therapy. Numerous studies have revealed that miRs are critical as tumor suppressors or oncogenes in osteosarcoma. However, their involvements in the underlying molecular mechanisms remain to be further elucidated. The present study revealed that the expression level of miR-506 was decreased in osteosarcoma tissues and cells compared with matched adjacent non-cancerous bone tissues and normal osteoblastic cells, respectively. In addition, an overexpression of miR-506 was revealed to suppress osteosarcoma cell proliferation and enhance apoptosis in vitro, and inhibit 


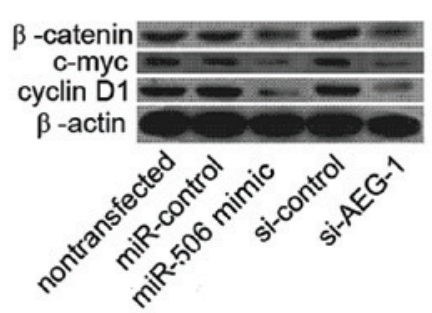

C

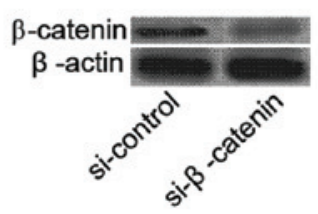

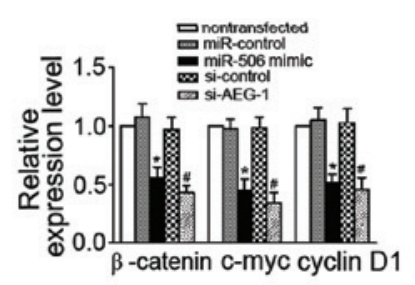

B

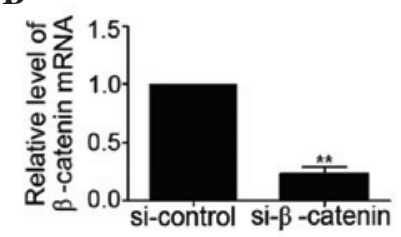

E

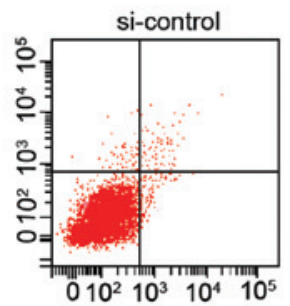

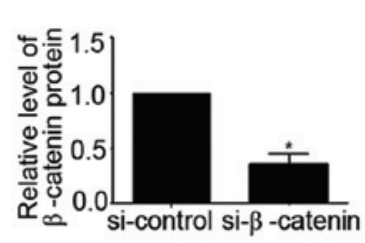

D

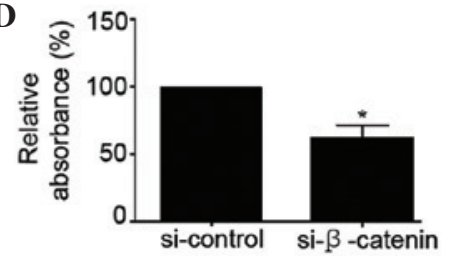

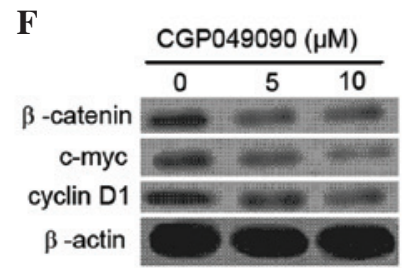

F

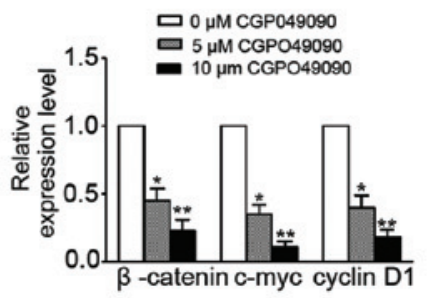

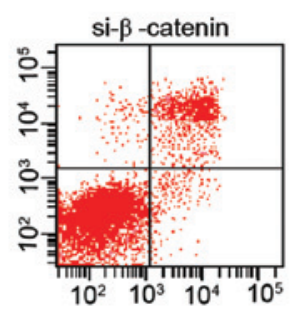

G

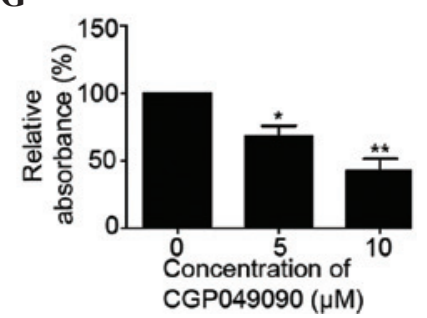

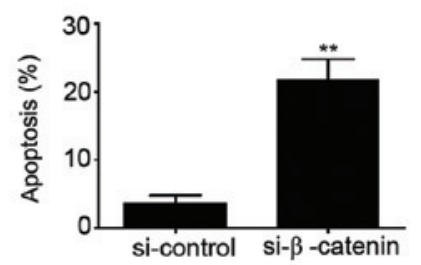

H

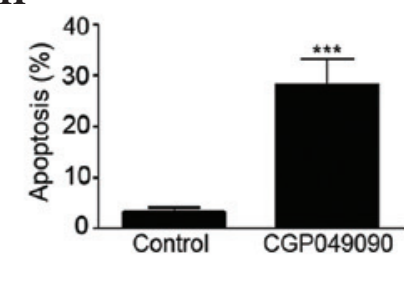

Figure 6. miR-506 inhibited osteosarcoma by targeting AEG-1 via the Wnt/ $\beta$-catenin pathway signaling. (A) Western blot analysis showed that overexpression of miR-506 and knockdown of AEG-1 decreased the expression levels of $\beta$-catenin (94 kDa), c-myc (49 kDa) and cyclin D1 (34 kDa) in human osteosarcoma MG63 cells. ${ }^{*} \mathrm{P}<0.05$ vs. miR-control or nontransfected; ${ }^{*} \mathrm{P}<0.05$ vs. si-control. (B and $\left.\mathrm{C}\right)$ Relative levels of $\beta$-catenin $(\mathrm{B}) \mathrm{mRNA}$ and $(\mathrm{C})$ protein were significantly decreased in MG63 cells transfected with si- $\beta$-catenin. ${ }^{*} \mathrm{P}<0.05,{ }^{* *} \mathrm{P}<0.01$ vs. si-control. (D and E) Blocking the Wnt/ $\beta$-catenin pathway using si- $\beta$-catenin (D) inhibited MG63 cell proliferation and (E) induced apoptosis of MG63 cells. ${ }^{*} \mathrm{P}<0.05,{ }^{* *} \mathrm{P}<0.01$ vs. si-control. (F-H) CGP049090, a small molecule inhibitor of Wnt/ $\beta$-catenin, $(\mathrm{F})$ inhibited the expression of $\beta$-catenin, c-myc and cyclin D1 in MG63 cells in a concentration-dependent manner, (G) inhibited MG63 cell proliferation in a concentration-dependent manner and (H) induced apoptosis of MG63 cells $\left(10 \mu \mathrm{M} \mathrm{CGP049090).}{ }^{*} \mathrm{P}<0.05,{ }^{* *} \mathrm{P}<0.01\right.$,

*** $\mathrm{P}<0.001$ vs. control (0 $\mu \mathrm{M}$ CGP049090). miR, microRNA; AEG-1, astrocyte elevated gene-1; si, small interfering RNA.

tumor growth in vivo. The present data suggest that miR-506 is associated with the development and progression of osteosarcoma, and may be a promising diagnostic biomarker and therapeutic target for osteosarcoma. Recently, a study revealed that the miR-29 family are important in the development and progression of human osteosarcoma; serum levels of miR-29a and miR-29b were independent prognostic factors for overall and disease-free survival (9). Other studies have revealed that detection of serum miR-133b, miR-206, miR-148a, miR-196a and miR-196b expression has clinical potential as novel diagnostic biomarkers and are efficient predictors of prognosis in osteosarcoma patients $(27,28)$. Nevertheless, the carcinogenic mechanisms of these miRs on osteosarcoma have not been fully elucidate. Therefore, the present findings provide valuable information and therapeutic benefits in osteosarcoma development.

AEG-1 is known to be involved in multiple human cancers (18). Elevated AEG-1 expression is linked to progression of cervical intraepithelial neoplasia and poor prognosis in cervical cancer (29). Knockdown of AEG-1 induced prostate cancer cell apoptosis via the activation of forkhead box $3 a$ (30). In the present study, to investigate whether the loss function of AEG-1 is connected to the development of osteosarcoma, the expression of AEG-1 was downregulated in MG63 cells. The results indicated that knockdown of AEG-1 inhibited cell proliferation, suppressed colony-forming ability and induced apoptosis. In summary, the present data demonstrates that AEG-1 is involved in mediating cell proliferation and survival. Similarly, a previous study has revealed that AEG-1 regulates the migration and invasion of osteosarcoma U2OS cells (22). Furthermore, the present study confirmed that the 3'-UTR of AEG-1 is a functional target site for miR-506 in MG63 cells. Studies in other types of cancer have indicated that miR-506 has an antineoplastic function $(14,31)$; however, the role of miR-506 in tumor cells is not fully understood. A previous study indicated that downregulation of miR-506 in ovarian carcinoma facilitated an aggressive phenotype, whereas overexpression of miR-506 in ovarian cancer cells inhibited cell 
proliferation and promoted senescence by directly targeting the cyclin-dependent kinase 4/6-forkhead box M1 axis (32). miR-506 also represents a novel class of miR that regulates E-cadherin and vimentin/N-cadherin in the suppression of epithelial-to-mesenchymal transition and metastasis, and is associated with a good prognosis in epithelial ovarian cancer (31). To the best of our knowledge, the current results present the first evidence that miR-506 may have therapeutic potential against osteosarcoma.

The canonical Wnt/ $\beta$-catenin signaling pathway is one of the fundamental mechanisms that regulates cell proliferation, polarity and cell fate determination during embryonic development and homeostatic self-renewal in multiple adult tissues (33). Therefore, mutations in this pathway are often associated with cancer and other diseases. The Wnt $/ \beta$-catenin signaling pathway has been revealed to be excessively activated in osteosarcoma and contributes to the development and progression of osteosarcoma $(34,35)$. Dihydroartemisinin inhibits tumor growth of human osteosarcoma cells by elevating the catalytic activity of glycogen synthase kinase $3 \beta$ (GSK3 $\beta$ ), which results in lower protein level and transcriptional activity of $\beta$-catenin (36). Overexpression of bone morphogenetic protein 9 decreased the expression levels of $\beta$-catenin mRNA and protein, downregulated its downstream proteins c-myc and osteoprotegerin, and suppressed the phosphorylation level of GSK-3 $\beta$ (Ser 9) in osteosarcoma cells (37). In addition, a previous study revealed that celecoxib, a cyclooxygenase (COX)-2 inhibitor, exerted an inhibitory effect on the viability of MG63 cells in a time- and dose-dependent manner, by inhibiting the expression of $\beta$-catenin and c-myc, and encoding cyclin D1 (38). This suggests that $\beta$-catenin is required for MG63 cell survival and the $\mathrm{Wnt} / \beta$-catenin pathway is a COX-2-independent target for non-steroidal anti-inflammatory drugs in osteosarcoma. The present study revealed that the Wnt/ $\beta$-catenin signaling pathway was suppressed by overexpression of miR-506 or downregulation of AEG-1. Additionally, inhibition of the Wnt/ $\beta$-catenin signaling pathway by si- $\beta$-catenin or CGP049090 significantly attenuated the viability and evoked apoptosis of MG63 cells in the present study. Therefore, the present study suggests that AEG-1 promotes osteosarcoma development by activating the $\mathrm{Wnt} / \beta$-catenin pathway, and miR-506 downregulates the expression of AEG-1, which inhibits the $\mathrm{Wnt} / \beta$-catenin pathway and provides therapeutic benefits in osteosarcoma.

To conclude, the present study has demonstrated that overexpression of miR-506 suppresses proliferation and induces apoptosis of osteosarcoma cells by targeting AEG-1. In addition, the present study revealed that miR-506 exhibits antineoplastic abilities by regulating the Wnt/ $\beta$-catenin pathway. These findings provide novel insights for miRs in osteosarcoma.

\section{References}

1. Zhu J, Feng Y, Ke Z, Yang Z, Zhou J, Huang X and Wang L: Down-regulation of miR-183 promotes migration and invasion of osteosarcoma by targeting Ezrin. Am J Pathol 180: 2440-2451, 2012.

2. Huang J, Ni J, Liu K, Yu Y, Xie M, Kang R, Vernon P, Cao L and Tang D: HMGB1 promotes drug resistance in osteosarcoma. Cancer Res 72: 230-238, 2012.
3. Huang J, Liu K, Yu Y, Xie M, Kang R, Vernon P, Cao L, Tang D and Ni J: Targeting HMGB1-mediated autophagy as a novel therapeutic strategy for osteosarcoma. Autophagy 8: 275-277, 2012.

4. Chang E, Kim L, Choi JM, Park SE, Rhee EJ, Lee WY, Oh KW, Park SW, Park DI and Park CY: Ezetimibe stimulates intestinal glucagon-like peptide 1 secretion via the MEK/ERK pathway rather than dipeptidyl peptidase 4 inhibition. Metabolism 64: 633-641, 2015.

5. Liu M, Lang N, Qiu M, Xu F, Li Q, Tang Q, Chen J, Chen X, Zhang S, Liu Z, et al: miR-137 targets Cdc 42 expression, induces cell cycle G1 arrest and inhibits invasion in colorectal cancer cells. Int J Cancer 128: 1269-1279, 2011.

6. Chen L, Wang X, Wang H, Li Y, Yan W, Han L, Zhang K, Zhang J, Wang Y, Feng Y, et al: miR-137 is frequently down-regulated in glioblastoma and is a negative regulator of Cox-2. Eur J Cancer 48: 3104-3111, 2012.

7. Croce C: Introduction to the role of microRNAs in cancer diagnosis, prognosis and treatment. Cancer J 18: 213-214, 2012.

8. Zhang C, Yao C, Li H, Wang G and He X: Combined elevation of microRNA-196a and microRNA-196b in sera predicts unfavorable prognosis in patients with osteosarcomas. Int J Mol Sci 15: 6544-6555, 2014.

9. Hong Q, Fang J, Pang Y and Zheng J: Prognostic value of the microRNA-29 family in patients with primary osteosarcomas. Med Oncol 31: 37, 2014.

10. Liu LH, Li H, Li JP, Zhong H, Zhang HC, Chen J and Xiao T: miR-125b suppresses the proliferation and migration of osteosarcoma cells through down-regulation of STAT3. Biochem Bioph Res Commun 416: 31-38, 2011.

11. Osaki M, Takeshita F, Sugimoto Y, Kosaka N, Yamamoto Y, Yoshioka Y, Kobayashi E, Yamada T, Kawai A, Inoue T, et al: MicroRNA-143 regulates human osteosarcoma metastasis by regulating matrix metalloprotease-13 expression. Mol Ther 19: 1123-1130, 2011.

12. Duan Z, Choy E, Harmon D, Liu X, Susa M, Mankin H and Hornicek F: MicroRNA-199a-3p is downregulated in human osteosarcoma and regulates cell proliferation and migration. Mol Cancer Ther 10: 1337-1345, 2011.

13. Zhao Y, Liu H, Li Y, Wu J, Greenlee AR, Yang C and Jiang Y: The role of miR-506 in transformed 16HBE cells induced by anti-benzo[a]pyrene-trans-7, 8-dihydrodiol-9, 10-epoxide. Toxicol Lett 205: 320-326, 2011.

14. Arora H, Qureshi R and Park WY: miR-506 regulates epithelial mesenchymal transition in breast cancer cell lines. PloS One 8: e64273, 2013.

15. Tong JL, Zhang CP, Nie F, Xu XT, Zhu MM, Xiao SD and Ran ZH: MicroRNA 506 regulates expression of PPAR alpha in hydroxycamptothecin-resistant human colon cancer cells. FEBS Lett 585: 3560-3568, 2011.

16. He W, He S, Wang Z, Shen H, Fang W, Zhang Y, Qian W, Lin M, Yuan J, Wang J, et al: Astrocyte elevated gene-1 (AEG-1) induces epithelial-mesenchymal transition in lung cancer through activating Wnt/ $\beta$-catenin signaling. BMC Cancer 15: 107, 2015.

17. Emdad L, Sarkar D, Su ZZ, Randolph A, Boukerche H, Valerie K and Fisher PB: Activation of the nuclear factor kappaB pathway by astrocyte elevated gene-1: Implications for tumor progression and metastasis. Cancer Res 66: 1509-1516, 2006.

18. Yoo BK, Emdad L, Su ZZ, Villanueva A, Chiang DY, Mukhopadhyay ND, Mills AS, Waxman S, Fisher RA, Llovet JM, et al: Astrocyte elevated gene-1 regulates hepatocellular carcinoma development and progression. J Clin Invest 119: 465-477, 2009.

19. Yu C, Chen K, Zheng H, Guo X, Jia W, Li M, Zeng M, Li J and Song L: Overexpression of astrocyte elevated gene-1 (AEG-1) is associated with esophageal squamous cell carcinoma (ESCC) progression and pathogenesis. Carcinogenesis 30: 894-901, 2009.

20. Liu B, Wu Y and Peng D: Astrocyte elevated gene-1 regulates osteosarcoma cell invasion and chemoresistance via endothelin-1/endothelin A receptor signaling. Oncol Lett 5: 505-510, 2013.

21. Wang F, Ke Z-F, Sun SJ, Chen WF, Yang SC, Li SH, Mao XP and Wang LT: Oncogenic roles of astrocyte elevated gene-1 (AEG-1) in osteosarcoma progression and prognosis. Cancer Biol Ther 12: 539-548, 2011

22. Wang F, Ke ZF, Wang R, Wang YF, Huang LL and Wang LT: Astrocyte elevated gene-1 (AEG-1) promotes osteosarcoma cell invasion through the JNK/c-Jun/MMP-2 pathway. Biochem Bioph Res Commun 452: 933-939, 2014.

23. Schajowicz F (ed): World Health Organization: Histological Typing of Bone Tumours. 2nd edition. Springer-Verlag, Berlin, 1993. 
24. Livak KJ and Schmittgen TD: Analysis of relative gene expression data using real-time quantitative PCR and the 2(-Delta Delta C(T)) Method. Methods 25: 402-408, 2001.

25. Liu H, Song X, Liu C, Xie L, Wei L and Sun R: Knockdown of astrocyte elevated gene-1 inhibits proliferation and enhancing chemo-sensitivity to cisplatin or doxorubicin in neuroblastoma cells. J Exp Clin Cancer Res 28: 19, 2009.

26. Guan H, Tan P, Xie L, Mi B, Fang Z, Li J, Yue J, Liao H and Li F: FOXO1 inhibits osteosarcoma oncogenesis via Wnt/ $\beta$-catenin pathway suppression. Oncogenesis 4: e166, 2015

27. Zhang C, Yao C, Li H, Wang G and He X: Serum levels of microRNA-133b and microRNA-206 expression predict prognosis in patients with osteosarcoma. Int J Clin Exp Pathol 7: 4194-4203, 2014.

28. Ma W, Zhang X, Chai J, Chen P, Ren P and Gong M: Circulating miR-148a is a significant diagnostic and prognostic biomarker for patients with osteosarcoma. Tumor Biol 35: 12467-12472, 2014.

29. Huang K, Li LA, Meng Y, You Y, Fu X and Song L: High expression of astrocyte elevated gene-1 (AEG-1) is associated with progression of cervical intraepithelial neoplasia and unfavorable prognosis in cervical cancer. World J Surg Oncol 11: 297, 2013.

30. Kikuno N, Shiina H, Urakami S, Kawamoto K, Hirata H, Tanaka Y, Place RF, Pookot D, Majid S, Igawa M and Dahiya R: Knockdown of astrocyte-elevated gene-1 inhibits prostate cancer progression through upregulation of FOXO3a activity. Oncogene 26: 7647-7655, 2007.

31. Sun Y, Hu L, Zheng H, Bagnoli M, Guo Y, Rupaimoole R, Rodriguez-Aguayo C, Lopez-Berestein G, Ji P, Chen K, et al: MiR-506 inhibits multiple targets in the epithelial-to-mesenchymal transition network and is associated with good prognosis in epithelial ovarian cancer. J Pathol 235: 25-36, 2015.
32. Liu G, Sun Y, Ji P, Li X, Cogdell D, Yang D, Parker Kerrigan BC, Shmulevich I, Chen K, Sood AK, et al: MiR-506 suppresses proliferation and induces senescence by directly targeting the CDK4/6-FOXM1 axis in ovarian cancer. J Pathol 233: 308-318, 2014.

33. MacDonald BT, Tamai K and He X: Wnt/beta-catenin signaling: Components, mechanisms, and diseases. Dev Cell 17: 9-26, 2009.

34. Ma Y, Ren Y, Han EQ, Li H, Chen D, Jacobs JJ, Gitelis S, O'Keefe RJ, Konttinen YT, Yin G and Li TF: Inhibition of the Wnt- $\beta$-catenin and Notch signaling pathways sensitizes osteosarcoma cells to chemotherapy. Biochem Bioph Res Commun 431: 274-279, 2013.

35. Hoang BH, Kubo T, Healey JH, Yang R, Nathan SS, Kolb EA, Mazza B, Meyers PA and Gorlick R: Dickkopf 3 inhibits invasion and motility of Saos-2 osteosarcoma cells by modulating the Wnt-beta-catenin pathway. Cancer Res 64: 2734-2739, 2004.

36. Liu Y, Wang W, Xu J, Li L, Dong Q, Shi Q, Zuo G, Zhou L, Weng Y, Tang M, et al: Dihydroartemisinin inhibits tumor growth of human osteosarcoma cells by suppressing Wnt $/ \beta$-catenin signaling. Oncol Rep 30: 1723-1730, 2013.

37. Lv Z, Wang C, Yuan T, Liu Y, Song T, Liu Y, Chen C, Yang M, Tang Z, Shi Q and Weng Y: Bone morphogenetic protein 9 regulates tumor growth of osteosarcoma cells through the Wnt// 3 -catenin pathway. Oncol Rep 31: 989-994, 2014.

38. Xia JJ, Pei LB, Zhuang JP, Ji Y, Xu GP, Zhang ZP, Li N and Yan JL: Celecoxib inhibits $\beta$-catenin-dependent survival of the human osteosarcoma MG-63 cell line. J Int Med Res 38: 1294-1304, 2010. 